The Astrophysical Journal, 677:488-494, 2008 April 10

(C) 2008. The American Astronomical Society. All rights reserved. Printed in U.S.A.

\title{
A MORPHOLOGICAL APPROACH TO THE PULSED EMISSION FROM SOFT GAMMA REPEATERS
}

\author{
J. J. JIA, ${ }^{1,2}$ Y. F. HuANG, ${ }^{1,3}$ AND K. S. CHENG ${ }^{1}$ \\ Received 2007 August 26; accepted 2008 January 9
}

\begin{abstract}
We present a geometrical methodology to interpret the periodical light curves of soft gamma repeaters based on the magnetar model and the numerical arithmetic of the three-dimensional magnetosphere model for the young pulsars. The hot plasma released by the starquake is trapped in the magnetosphere, and photons are emitted tangentially to the local magnetic field lines. The variety of radiation morphologies in the burst tails and the persistent stages could be well explained by the trapped fireballs on different sites inside the closed field lines. Furthermore, our numerical results suggest that the pulse profile evolution of SGR 1806-20 during the 2004 December 27 giant flare is due to a lateral drift of the emitting region in the magnetosphere.
\end{abstract}

Subject headings: gamma rays: bursts - stars: magnetic fields — stars: neutron - X-rays: stars

\section{INTRODUCTION}

Soft Gamma Repeaters (SGRs) seemed weird since the first discovery on 1979 March 5 (Mazets et al. 1979) for their mysterious characteristics such as the large energy release, the repetitive emission of bursts in hard X-rays or soft $\gamma$-ray bands, and the pulsed periodical emissions after the bursts and in the quiescent stages whose morphologies are both energy-dependent and timedependent (see Woods \& Thompson 2004 for a recent review). So far, the catalog ${ }^{4}$ has four SGRs confirmed plus one candidate. SGRs are found to be associated with young $\left(\sim 10^{4} \mathrm{yr}\right)$ supernova remnants (SNRs), and their spin periods are $\sim 5-8 \mathrm{~s}$, at a large spin-down rate of about $10^{-11} \mathrm{~s} \mathrm{~s}^{-1}$, which gives an inferred ultrastrong dipolar magnetic field on the order of $\sim 10^{14}-10^{15} \mathrm{G}$.

A variety of models were proposed to understand the physics of SGRs, and it is now widely accepted that SGRs are neutron stars with a magnetic field of $\sim 10^{14}-10^{15} \mathrm{G}$ (Thompson \& Duncan 1995, 1996). Unlike most of the pulsars in the neutron star family, which are powered by their spin-down, the high-luminosity bursts and the persistent $X$-ray or soft $\gamma$-ray pulsations of magnetars come from the decay of their ultrastrong magnetic fields. The spectrum of the persistent X-ray emission could be fitted by a superposition of a blackbody component and a power law, which suggests that besides the radiation from the neutron star surface, there is another component coming from the magnetosphere.

The high-luminosity burst has now been successfully interpreted by the dissipation of magnetic energy. However, the persistent long-period pulsations in the quiescent $\mathrm{X}$-ray emission of SGRs are still not well understood, because of their complicated and astonishing morphologies. Thompson et al. (2002) assumed that the angular pattern of the X-ray flux is modified by the resonant cyclotron scattering at a distance about $50-100 \mathrm{~km}$ above the neutron star, and multipulses are generated by some effects associated with the twisted magnetosphere (e.g., the optical depth is thin near two magnetic poles, and thick at the magnetic equator). However, their numerical results from the Monte Carlo simulation has a bias in favor of the orthogonal dipole (i.e., the magnetic axis is perpendicular to the spin axis), and the viewing angle has to also be

\footnotetext{
1 Department of Physics, University of Hong Kong, Pokfulam Road, Hong Kong, China.

2 Department of Physics and Astronomy, Johns Hopkins University, Baltimore, MD 21218

3 Department of Astronomy, Nanjing University, Nanjing 210093, China.

4 See http://www.physics.mcgill.ca/ pulsar/magnetar/main.html.
}

nearly $90^{\circ}$ from the spin axis, for the photons escaping from optically thin poles (Fernández \& Thompson 2007). In addition, the separation of the strong peaks in their calculations is always $\pi$ (halfphase cycle), which differs from the observations. C. Thompson (2005, private communication) agreed that the change in the persistent pulse profile reflects a redistribution of persistent currents on closed field lines. However, he ascribed such redistribution to the gradual change in the magnetic twist at a distance of $30-100 \mathrm{~km}$, while our understanding is that the redistribution is due to the gradual migration of the emitting regions (crustal platelet motion or hot plasma drifting in the magnetosphere-we will discuss the possibility of each candidate in $\S 4$ ).

In this paper, we present some simulated pulsed profiles by assuming that the radiation region is located in the closed field lines, and make attempts to simulate the radiation morphology evolution in one particular event, i.e., the SGR 1806-20 burst on 2004 December 27. We first have a general review on the timing properties of SGRs in $\S 2$. In $\S 3$, we present the motivation to reproduce the light curves in the closed field lines and the resultant profiles. In $\S 4$, we apply the geometrical model to the well-known 2004 December burst of SGR 1806-20 and calculate the radiation morphology by a three-dimensional magnetosphere simulation. We also try to explain the change in the persistent pulse profile. A brief discussion is given in $\S 5$.

\section{PERIODICAL EMISSIONS FROM SGRs}

The periodical emissions of the four confirmed SGRs have been detected in the decay of the burst and the quiescent or persistent stages, with periods in the 5-8 s range, which are the spin periods of neutron stars. The pulse profiles show many interesting and even surprising morphologies, some of which are completely different from the canonical radio or high-energy pulsars. The characteristics of light curves of the X-ray and $\gamma$-ray pulsarssuch as number of peaks, the peak separation, and the relative amplitudes of the peaks - are unchangeable, while those of the SGRs are time dependent. Here, we list several main features of the persistent emissions from SGRs:

1. Multipeaked Morphology.-The most dramatic example is the pulse profile change of SGR 1900+14 after the outburst on 1998 August 27. A four-peaked repetitive pattern of the X-ray light curve was detected by both Ulysses and BeppoSAX half a minute after the burst onset (Hurley et al. 1999; Feroci et al. 2001 ), and these peaks were found to be evenly spaced at $1.0 \mathrm{~s}$ 
intervals on the $5.16 \mathrm{~s}$ rotation period. Several minutes later, this multipeaked profile evolved as a simple sinusoidal morphology (Woods \& Thompson 2004), and this evolution in pulse profile lasted for a couple of years. Such change in morphology is also found in the RXTE PCA archive of SGR 1806-20 between 1996 and 2005 (Woods et al. 2007). During the 10 year observation, the $2-10 \mathrm{keV}$ pulse shape evolved from one broad peak pattern to a three-peaked one in 2003, and then to the sinusoidal shape again until the multipeaked profile after the burst on 2004 December (see Fig. 3 in Woods et al. 2007).

2. Relative Magnitudes of Peaks.-Besides the evolution of the number of peaks, the relative magnitudes of the peaks in one phase cycle also change with time. Palmer et al. (2005) showed one such pulse profile evolution during the giant flare of 2004 December 27. The folded light curves in different time intervals from 30 to $265 \mathrm{~s}$ following the main spike indicate the growth of the second peak, whose intensity related to the primary peak increases from the DC level to nearly equal in height. In the other words, we can say that the primary peak fades until it reaches the same magnitude as the secondary one. At the late stage of the decay of the giant flare, the relative magnitude of the third peak, 0.2 in the phase prior to the primary one, starts to grow larger.

3. Energy-Dependent Profiles.-The evolution of pulse profiles of SGRs is not only time-dependent, but also changes in different energy bands. Woods et al. (2007) investigated the energy dependence of the SGR 1806-20 pulse profiles in three energy bands between 2 and $40 \mathrm{keV}$. Six months before the flare, there was only one broad peak in the pulse profile below $15 \mathrm{keV}$, and it showed two clear peaks in the $15-40 \mathrm{keV}$ band. After the giant flare, the light curve became more complicated, showing multiple peaks in all energy intervals, and the peaks were inconsistent in phase (see Fig. 4 in Woods et al. 2007).

These completely different phenomena require completely different physics in this small (or maybe not small) group of neutron stars, compared with the canonical pulsars. The thermal spectrum component suggests that the radiation partly comes from the hot spots on the stellar surface. In addition, the phase inconsistence of the pulse profile indicates that it may not be localized in a particular region, and the unpredictable starquakes can produce the randomly localized emitting regions during every burst event. This is the main motivation for us to build up the model of the alterable pulse profiles in $\S 3$.

\section{RADIATIONS FROM THE CLOSED FIELD LINES}

\subsection{Why Closed Regions?}

The theoretical models for radiation from high-energy pulsars (e.g., Crab and Vela) require the radiation engine to be located in the open field line region in the polar gap model (Harding 1981; Daugherty \& Harding 1996), the outer gap model (Cheng et al. 1986; Chiang \& Romani 1994; Romani \& Yadigaroglu 1995; Cheng et al. 2000), and the modified outer gap model (Dyks \& Rudak 2003; Jia et al. 2007). However, we cannot apply these models to magnetars to explain their persistent X-ray emissions. We assume that the pulsed emissions of SGRs in the decay or afterglow of the bursts are originating in the closed regions rather than in the open regions. We have three main reasons for confining the radiation regions to the closed zones in the neutron star magnetosphere:

1. The volume occupied by the open field lines is much smaller than that of the closed field lines. Take SGR 1806-20, for example; the spin period is $P=7.56 \mathrm{~s}$, which means the radius of the light cylinder reaches $R_{L}=c P /(2 \pi)=3.6 \times 10^{10} \mathrm{~cm}$, where

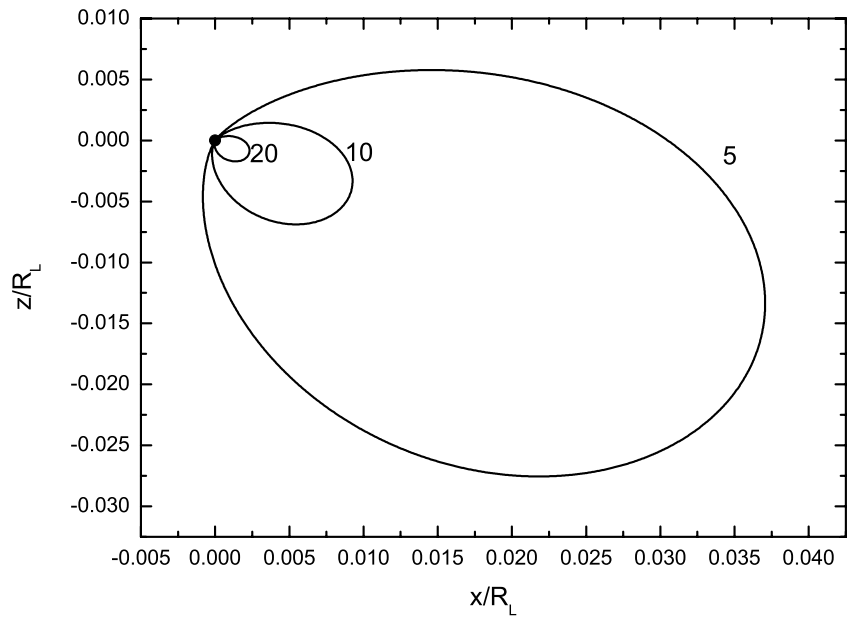

FIG. 1.-Closed field lines on the $\Omega$ - $\mu$ plane with layer parameters $a_{1}=5,10$, and 20. The inclination angle is $\alpha=30^{\circ}$, and the spin period of SGR 1806-20 $(P=7.56 \mathrm{~s})$ is applied. The neutron star is located at the point $(0,0)$.

$c$ is the speed of light. Thus, the radius of the polar cap is $R_{p}=$ $R_{*}\left(R_{*} / R_{L}\right)^{1 / 2}=5.3 \times 10^{3} \mathrm{~cm}$, corresponding to an angular size of $\theta_{p}=0.3^{\circ}$, much smaller than the characteristic size of the crustal platelet, where $R_{*}=10^{6} \mathrm{~cm}$ is the stellar radius. So, the polar cap area is only $10^{-5}$ of the neutron star surface, and there is no reason why the crustal platelet, where the magnetic energy is released, should be located in the open area.

2. The open field lines reach the light cylinder, where the corotating speed approaches the speed of light, and relativistic effects play a significant role in the radiation morphology (Romani \& Yadigaroglu 1995). Such effects lead to the sharp and narrow peaks of the light curves, and both peaks are produced by a single pole. However, those effects become less important when they are applied to the closed field lines, for they are much closer to the stellar surface than the open lines. As shown in Figure 1, we find that the farthest distance the closed field lines can reach drops much more quickly when their footprints are displaced further away from the polar cap. For the closed line on the plane (on which both the magnetic axis and spin axis lie) with layer parameter $a_{1}=5$, it only reaches a distance less than $0.05 R_{L}$ away from the stellar surface, and $0.01 R_{L}$ for $a_{1}=10$ (the definition of $a_{1}$ is given in $\S 3.2$ ). Thus, the double-peaked profiles are not necessary for closed field lines, and the broad peak could be a general product instead of the sharp one. Other features, like the peak separation and the multipeaks, could also be explained by the closed field lines (details to be discussed in $\S 3.2$ ).

3. The emitting region need not be localized on any particular site on the neutron star surface or inside the magnetosphere, while in the open field lines, the accelerating gap is restricted to the null charge surface (Goldreich \& Julian 1969; Cheng et al. 2000). This freedom in loci ensures the variety of the pulse profiles during different outbursts, for we can argue that time-dependent light curves result from different bunches of magnetic field lines where the plasma is trapped.

\subsection{Strategies for Numerical Simulation}

Since we argue that the periodic pulsed radiation of magnetars in the quiescent state is released from closed field line regions rather than open ones, we could apply the 3D magnetosphere model (Cheng et al. 2000) to simulate the pulse profiles. The boundary of these two different regions is defined as a group of so called lastclosed or first-open magnetic field lines, which are tangential to 
the light cylinder. The hot plasma is trapped in the closed region and oscillates along the closed field lines, and photons are assumed to be emitted outwardly along the tangential direction of the magnetic field lines, which makes the pulsed radiation much different from the one generated in the open area. In order to be consistent with the pulsar magnetosphere calculation in the high-energy pulsar models, we adopted the same definition for the coordinates of footprints of the magnetic field lines and layer parameters (Cheng et al. 2000). The shape of the polar cap could be determined by the footprints of the last-closed field lines anchored in the stellar surface, and we can label the coordinates of these footprints as $\left(x_{0}, y_{0}, z_{0}\right)$. Then we are able to define another set of footprints of magnetic field lines $\left(x_{0}^{\prime}, y_{0}^{\prime}, z_{0}^{\prime}\right)$ by multiplying a factor $a_{1}$, called the layer parameter: $x_{0}^{\prime}=a_{1} x_{0}, y_{0}^{\prime}=a_{1} y_{0}$, and $z_{0}^{\prime}=\left[1-\left(x_{0}^{\prime 2}+\right.\right.$ $\left.\left.y_{0}^{\prime 2}\right)\right]^{1 / 2}$, where $a_{1}>1$ indicates a closed region, and $a_{1}<1$ represents the open ones.

Since the magnetosphere is corotating with the neutron star, an aberration effect occurs along the line of sight. Thus, we have the aberrated emission direction (in the observer's frame) $\boldsymbol{u}^{\prime}=$ $\left(u_{r}^{\prime}, u_{\theta}^{\prime}, u_{\phi}^{\prime}\right)$ in the expression of the direction $\boldsymbol{u}=\left(u_{r}, u_{\theta}, u_{\phi}\right)$ in the corotating frame, and the rotational speed $\beta=|r \times Q| / c$ :

$$
\begin{aligned}
& u_{r}^{\prime}=\frac{u_{r} \sqrt{1-\beta^{2}}}{\left(1+\beta u_{\phi} c\right)}, \\
& u_{\theta}^{\prime}=\frac{u_{\theta} \sqrt{1-\beta^{2}}}{\left(1+\beta u_{\phi} c\right)}, \\
& u_{\phi}^{\prime}=\frac{u_{\phi}+\beta c}{\left(1+\beta u_{\phi} c\right)} .
\end{aligned}
$$

Another effect taken into account is the time of flight, which differs significantly, because the photons originated at different sites inside the magnetosphere. This effect can lead to the phase difference of the arrival photons comparable to the light curve period. Combing these two effects, and choosing the rotational axis as the $z$-axis, we obtain the phase angle $\Phi$ and the polar angle $\zeta$ given by (Yadigaroglu 1997)

$$
\begin{aligned}
\cos \zeta & =u_{z}^{\prime} / u^{\prime}, \\
\Phi & =-\phi_{u^{\prime}}^{\prime}-\boldsymbol{r} \cdot \hat{\boldsymbol{u}}^{\prime} / R_{L},
\end{aligned}
$$

where $\phi_{u^{\prime}}^{\prime}=\arccos \left(u_{x}^{\prime} / u_{x y}^{\prime}\right)$ [where $(x, y, z)$ is the Cartesian coordinate system] is the azimuthal angle in the observer's frame. Choosing $\Omega-\mu$ plane to be the $x-z$ plane, $u_{x y}^{\prime}$ is the length of the projection of $\hat{\boldsymbol{u}}^{\prime}$ on the $x-y$ plane.

\subsection{Simulated Light Curve Profiles}

In the following, we adopt the magnetic coordinates $\left(\theta_{*}, \phi_{*}\right)$ to describe the loci of radiation regions in the magnetosphere. The polar angle $\theta_{*}$ is defined as the angle with respect to the magnetic axis, instead of the rotational axis. The emitting region is assumed to be in the shape of a band along the azimuthal direction, with a relatively smaller longitudinal thickness compared with its azimuthal width. As the neutron star is rapidly rotating, we set the phase of the $\Omega-\boldsymbol{\mu}$ plane defined by the rotational axis and magnetic axis as $\phi_{*}=0^{\circ}$. Therefore, the transverse extension of the emitting region along the azimuthal direction could be expressed as $\Delta \phi_{*}$, which is treated as a parameter in our numerical simulations in this paper. However, there is no particular parameter for a quantitative longitudinal thickness $\Delta \theta_{*}$ in our simulation, and we represent it by the layer parameter $a_{1}$ instead.

Combining those factors mentioned above, we show in Figures 2 and 3 two typical light curves generated in the closed field
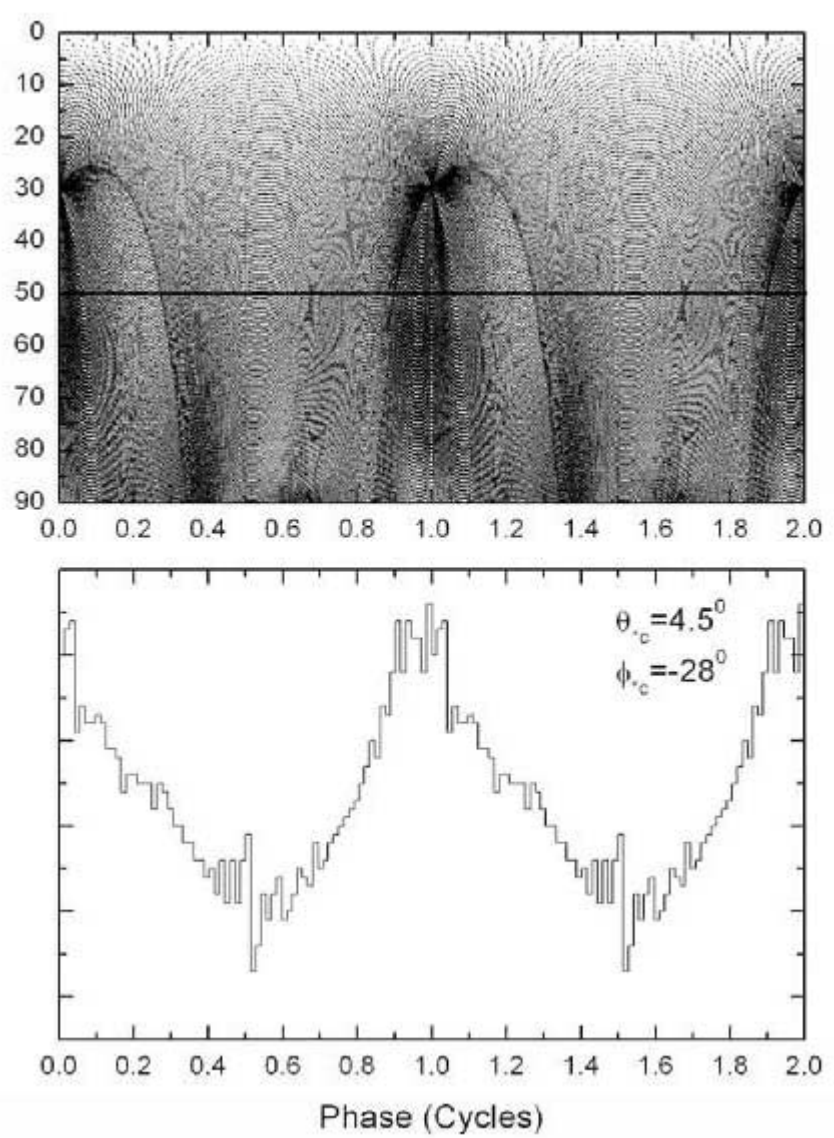

FIG. 2.-Top: Emission projections on the $(\zeta, \Phi)$-plane produced by the emitting region with azimuthal width of $\Delta \phi_{*}=180^{\circ}$ at layer $a_{1}=15$. The solid line indicates the line of sight at the viewing angle $\zeta=50^{\circ}$, and the darker regions correspond to greater intensities. Bottom: The single sinusoidal pattern of pulse profile corresponding to $\zeta=50^{\circ}$. SGR $1860-20$ parameters are used in the calculation, and other parameters for fitting are given in Table 1.

line zone. Figure 2 is a single sinusoidal pattern, and Figure 3 is a double-peaked morphology without any off-pulse phase. The spin period of SGR $1806-20$ is applied in the calculation, and the inclination angle and viewing angle with respect to the spin axis are chosen to be $30^{\circ}$ and $50^{\circ}$. Both pulse profiles are commonly detected in the SGRs' timing observations (e.g., Woods et al. 2007). The azimuthal widths of the emission regions for these two cases are $\Delta \phi_{*}=180^{\circ}$, and the other parameters for these two plots, like $a_{1}$ and the $\left(\theta_{*}, \phi_{*}\right)$ coordinates of the emitting regions, are given in Table 1 . In the upper panels of both figures, we show the emission projections onto the $(\zeta, \Phi)$-plane to illustrate the two kinds of light curves, respectively. We can find that the whole $(\zeta, \Phi)$-plane is fully filled by the emissions from closed field lines, compared with those partially filled by the outer gap in the open lines (e.g., Figs. 6-8 in Cheng et al. 2000). The emission projection of Figure 3 has a dense bundle in the phase range $(0.5,0.7)$, which produces the secondary peak of the doublepeaked light curve.

What makes such a difference between these two kinds of light curves? As shown by Cheng et al. (2000) the neutron star rotation results in a nonuniform distribution of the magnetic field lines in the magnetosphere, i.e., the magnetic field lines are swept back to accumulate around the $\boldsymbol{\Omega}-\boldsymbol{\mu}$ plane. In our calculation, the only parameter that is different in both cases is the longitude of the emitting region. On the side, around $\phi_{*}=0^{\circ}$, the accumulated field lines produce the one-peak dominated light curve profile (e.g., the single sinusoidal pattern), while on the other side, around 

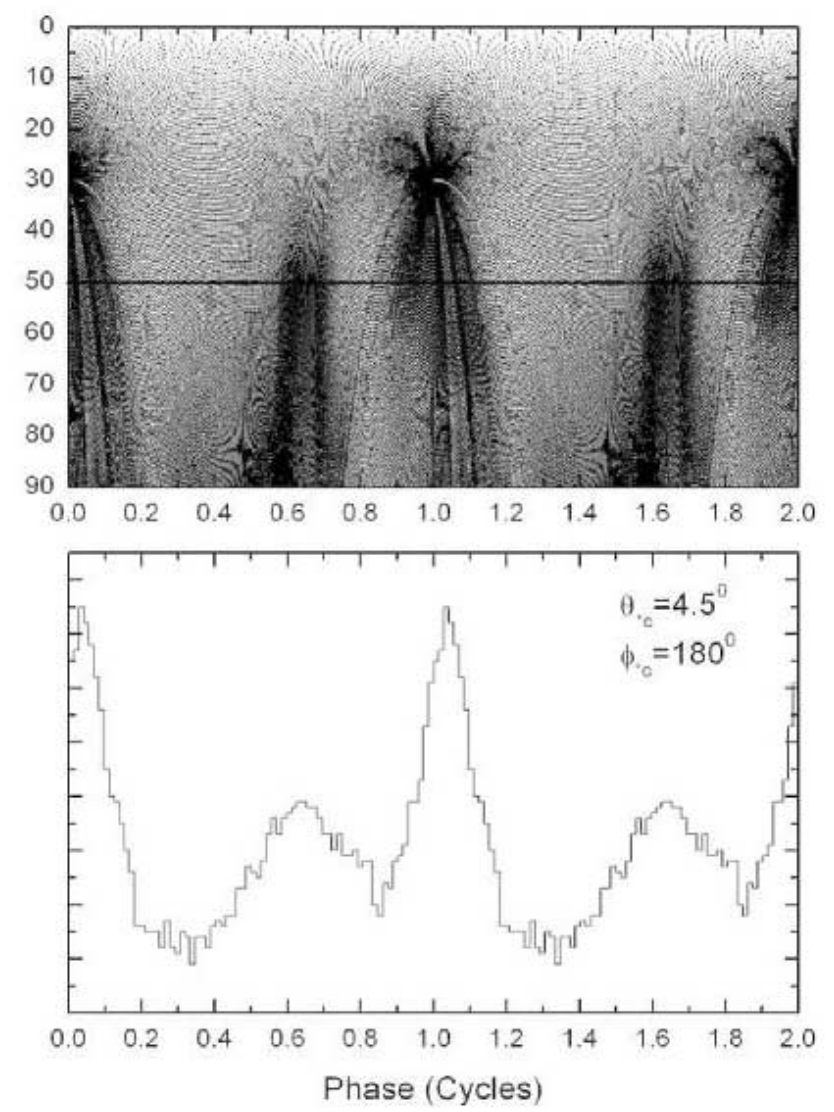

Fig. 3.-Top: Emission projections on the $(\zeta, \Phi)$-plane. The same fitting parameters as those in Fig. 2, except $\phi_{* c}$. Bottom: The double-peaked pattern of the pulse profile corresponding to $\zeta=50^{\circ}$.

$\phi_{*}=180^{\circ}$, the widely separated field lines make the doublepeaked profile possible.

\section{APPLICATION TO SGR 1806-20 IN 2004 DECEMBER}

\subsection{Pulse Profile Evolution}

As illustrated in the standard magnetar model, the crust of the magnetar breaks when the magnetostatic equilibrium in the lower crust can no longer be sustained and launches a hot fireball, which triggers the outburst of SGRs. The released energy comes from the reconnection of magnetic field lines in a crustal plate, which can be modeled as

$$
E=\frac{B^{2}}{8 \pi} l^{3},
$$

where $B$ is the magnetic field in the crust, and $l$ is the size of the crustal plate. The energy released by SGR 1806-20 in the 2004 December 27 burst is estimated to be as high as $\sim 10^{46} \mathrm{erg}$, and by substituting the inferred magnetic field strength of order $10^{15} \mathrm{G}$, we can estimate $l \sim 10^{5} \mathrm{~cm}$, which is about the thickness of the neutron star crust. A clump of electron-positron or electron-proton plasma is then ejected into the magnetosphere and trapped by the magnetic field lines anchored in this crustal platelet. The charged particles emitted by the hot plasma travel along the closed magnetic field lines and radiate photons. In the following emission morphology simulation, we will assume the emissivity along the field lines is uniform. However, the emission region along the azimuthal and polar directions have a finite characteristic dimension corresponding to $\sim 10^{5} \mathrm{~cm}$. We assumed that either the
TABLE 1

Parameter Sets in Figs. 2-5

\begin{tabular}{|c|c|c|c|c|}
\hline Plot Label & $\begin{array}{c}\text { Typical Layer } \\
\left(a_{1}\right)\end{array}$ & $\begin{array}{c}\theta_{* c} \\
(\operatorname{deg})\end{array}$ & $\begin{array}{c}\phi_{* c} \\
(\mathrm{deg})\end{array}$ & $\begin{array}{l}\Delta \Phi_{*} \\
(\operatorname{deg})\end{array}$ \\
\hline Fig. 2 & 15 & 4.5 & -28 & 180 \\
\hline Fig. 3 & 15 & 4.5 & 180 & 180 \\
\hline Fig. $4 a \ldots \ldots . . . . . . .$. & 10 & 3.0 & -11 & 180 \\
\hline Fig. $4 b$ & 10 & 3.0 & 0 & 180 \\
\hline Fig. $4 c \ldots \ldots \ldots . . . .$. & 10 & 3.0 & 7.5 & 180 \\
\hline Fig. $5 d \ldots \ldots \ldots \ldots$ & 20 & 6.0 & 7.5 & 180 \\
\hline
\end{tabular}

Notes.-Here, $\left(\theta_{* c}, \phi_{* c}\right)$ are the magnetic coordinates of the middle point of the emitting region, where $\theta_{* c}=a_{1} \theta_{p}$ is the product of the layer parameter and the angular radius of the polar cap, and $\phi_{* c}=0^{\circ}$ is defined as the $\Omega-\mu$ plane. Also, $\Delta \phi_{*}$ is the transverse extension of the emitting region along the azimuthal direction. The magnetic inclination angle is $\alpha=30^{\circ}$, and the viewing angel is $50^{\circ}$.

crustal motion driven by the neutron vortex (Ruderman 1991) or the lateral motion of the plasma across the field lines driven by the residual electric field, could lead to the change of the radiation morphologies. We will discuss which mechanism is more plausible later.

We attempted to simulate the pulse profile evolution during the giant flare of 2004 December 27 (e.g., Fig. 2 in Palmer et al. 2005). The most significant feature of the SGR 1806-20 pulsed radiation is the increased amplitude of the secondary peak related to the primary one. Our trials on the numerical simulation suggest that such change is due to the motion of emitting region. We present our results in Figure 4, which shows the effect of the azimuthal motion of the emitting region across the magnetic field lines with the typical layer parameter $a_{1}=10$, anchored in the crustal plate with the size about $1.5 \times 10^{5} \mathrm{~cm}$. Figure 4 also shows some other features (e.g., the width of the peaks and the phase separation between two peaks) to be consistent with the observation. The inclination angle of the magnetic dipole and the viewing angle of the observer are chosen to be $30^{\circ}$ and $50^{\circ}$, respectively. We assume that size of the plasma or the emitting region remains unchanged during the motion, and we describe the loci of the emitting region center in terms of $\left(\theta_{* c}, \phi_{* c}\right)$, which are the magnetic coordinates of the middle point of the emitting region at the typical layer. Since the motion is along the azimuthal direction, $\theta_{* c}=a_{1} \theta_{p}=3^{\circ}$ for all three panels in Figure 4 . As the $\boldsymbol{\Omega}-\boldsymbol{\mu}$ plane is defined as $\phi_{*}=0^{\circ}$, the azimuthal angle $\phi_{*}$ increases along the spin direction of the neutron star. The center of the radiation region was shifted from $\phi_{* c}=-11^{\circ}$ (Fig. $4 a$ ) to $\phi_{* c}=0^{\circ}$ (Fig. 4b), and finally at $\phi_{* c}=7.5^{\circ}$ (Fig. $4 c$ ) All parameters applied in our model fitting are listed in Table 1.

As shown by Arendt \& Eilek (1998) the polar cap of a rotating neutron star is asymmetrical and even probably discontinuous, which could make the radiation morphology complicated and asymmetrical. In our simulation, the primary peak at phase 0.9 of the light curve results from the majority of the magnetic field lines in which the plasma is trapped, and only a small portion of the field lines on the right edge of the plasma generate the secondary peak (at phase 1.2), where "right" means the site whose $\phi_{*}$ value is larger. As the plasma drifts from left to right (along the direction in which $\phi_{*}$ increases), more magnetic field lines at the right site are enrolled to radiate photons, which makes the secondary peak grow up. The radial distance of the local place where the arrival photons are generated is shown in Figure 6, below. Since the magnetosphere corotates with the neutron star, we calculate the modeling velocity of the emitting region motion in $\phi_{*}$-direction. As shown in Figure 7, below, the speed of the drift is estimated to be $\sim 10^{4} \mathrm{~cm} \mathrm{~s}^{-1}$ according to the pulse profile evolution timing by 

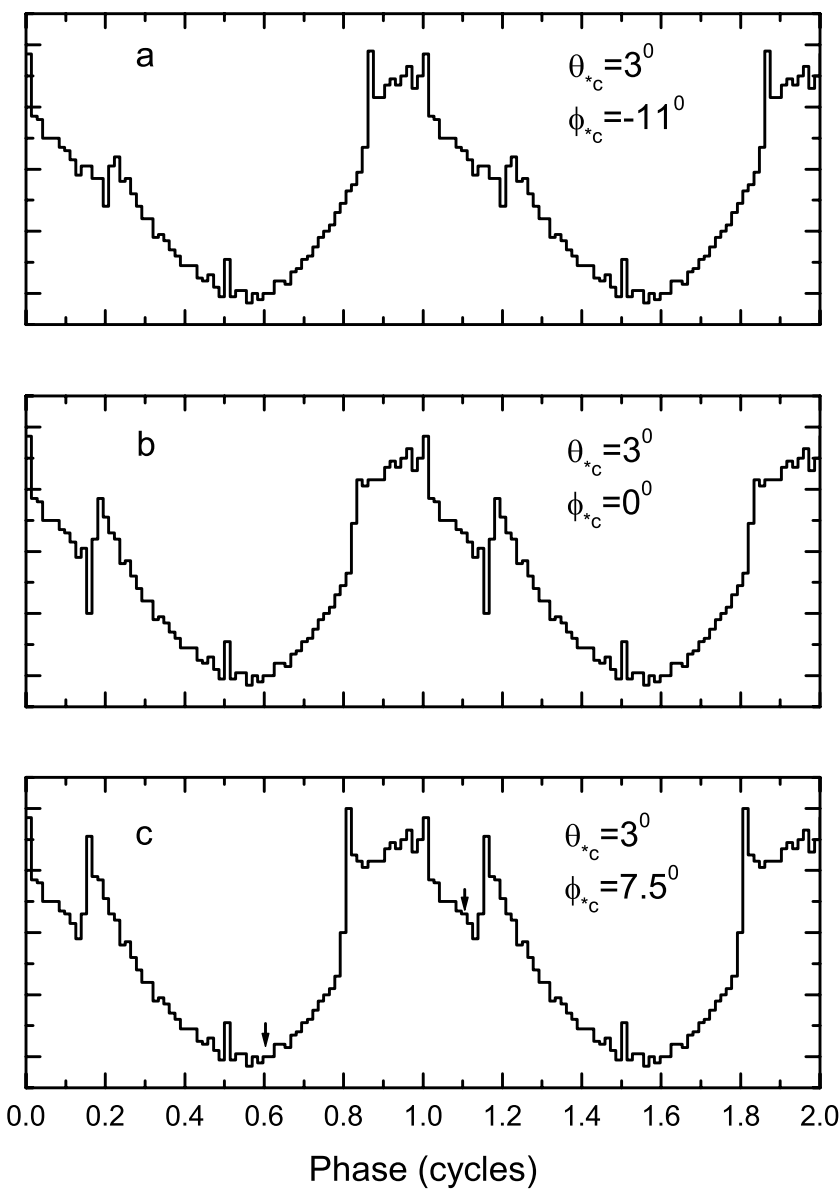

FIG. 4.- Simulated pulsed profiles of SGR 1806-20 in the giant flare on 2004 December 27. The emitting region drifts along the azimuthal direction, with the center coordinates indicated. The arrows in $(c)$ indicate the positions of the minor peaks of the radiation from both magnetic poles.

observation. We also give the expected radiation from the two magnetic poles in Figure $4 c$, which are indicated by two arrows. As the definition of the $(\Omega, \mu)$-plane, the magnetic poles are located at $\phi_{*}=0$ and $\pi$ ( 0 and 0.5 in the phase cycle), respectively. However, the time of flight effect makes the radiation from magnetic poles have a tiny shift in phase, e.g., 0.1 and 0.6. At the early stage, the flux of the radiation from the trapped plasma is so strong that the emissions from the polar caps could not be resolved. However, as the intensity of the two main peaks fades, the two minor peaks might become discriminable.

In general, the emitting region should not always move in one direction. Figure 5 shows the pulse profile evolution when the motion of the radiation region is changed to be along the $\theta$-direction. Figure $5 c$ is the same as Figure $4 c$, and the light curve of Figure $5 d$ is produced when the center of plasma is located at $a_{1}=20$, with the same azimuthal position as that in Figure $5 c$. We find that the morphology of the pulsed emission remains roughly unchanged and corresponds to the late stage ( $\sim 170 \mathrm{~s}$ after the burst) of the tail in the giant flare of 2004 December 27 . We want to remark that the light curve can also remain unchanged when the motion of the radiation region stops. We cannot differentiate these two possibilities from the light curve evolution.

\subsection{Interpretation of Numerical Results}

In order to simulate the time evolution of the light curves of SGR 1806-20, we have assumed that the emitting region can
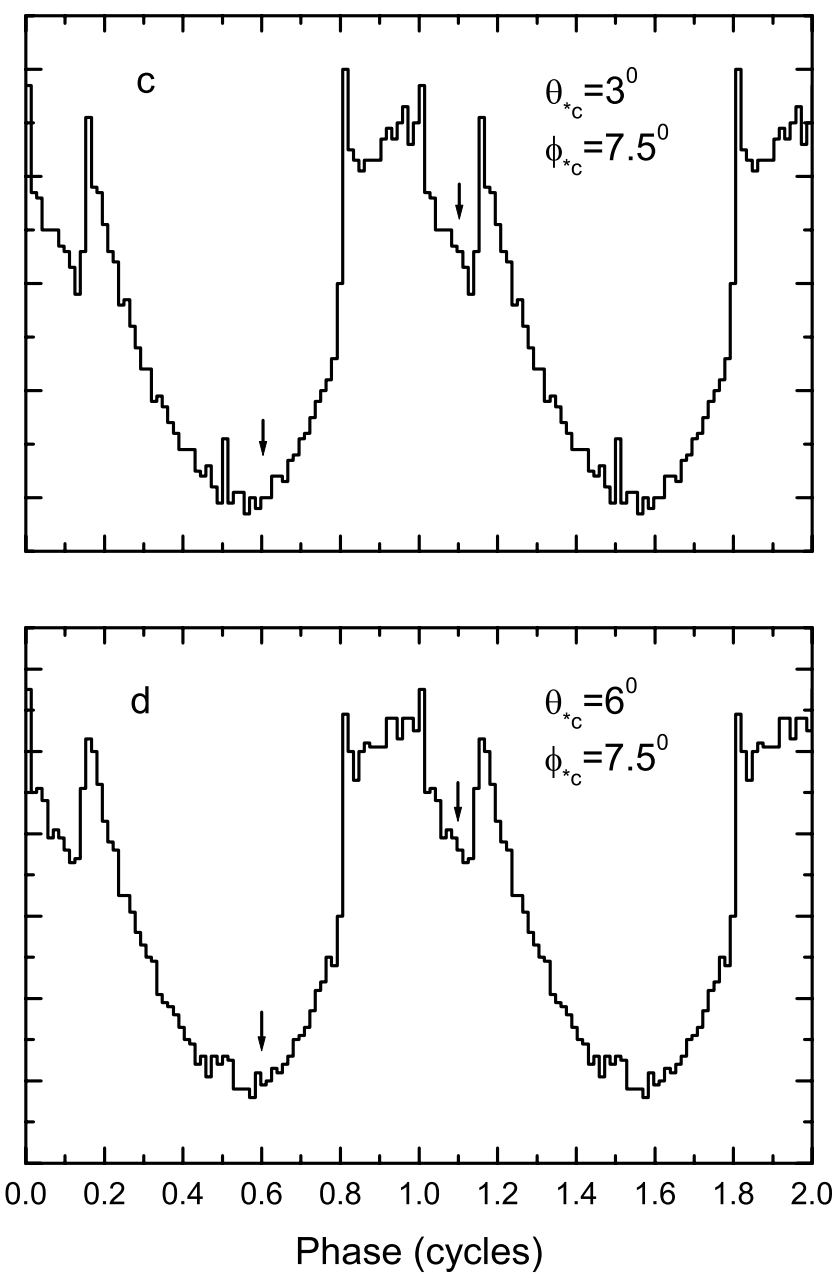

FIG. 5.- Simulated pulsed profiles of SGR 1806-20 in the giant flare on 2004 December 27. The arrows indicate the positions of the minor peaks of the radiation from both magnetic poles. The emitting region drifts in the direction perpendicular to that in Fig. 4.

migrate in the magnetosphere. In the following, we would like to discuss several possible movements induced in the crust and the magnetosphere, and their resultant speeds.

The interaction between the flux tubes and vortex lines in the core of neutron star make them interpin to each other. The vortex lines will move out of the core due to the spin-down of the star; they drag the flux tubes with them. However, the flux tubes are anchored in the crust; consequently, a large stress will be applied to the crust from the flux tubes (e.g., Ruderman 1991). When the crust breaks, the flux tubes will try to reduce their tension and drag the broken crust platelet. The maximum shear stress on the base of the crust from core magnetic flux tube motion which the crust could sustain is

$$
S \sim \frac{B B_{\text {crit }}}{8 \pi} \sim 3 \times 10^{29}{\text { dyn } \mathrm{cm}^{-2}}
$$

where $B_{\text {crit }} \sim 10^{16} \mathrm{G}$ is the critical magnetic field inside the magnetic flux tube. However, the crust may break before the magnetic stress reaches the maximum value. The shear modulus $\mu$ of a $1 \mathrm{~km}$ thick crust could not be larger than $10^{30} \mathrm{dyn}^{-2}$, and the crust may break when the stress reaches

$$
S_{\mathrm{brk}} \sim f \mu \theta_{\max }
$$


where $f$ is a factor of order unity and $\theta_{\max }$ is the yield strain under tension or compression, which is $\sim 10^{-1}-10^{-3}$ (e.g., Ruderman 1991).

Another mechanism to drive the crust to move is the vortex creeping. As the rotation of the neutron star slows down, the flux tubes are driven outwardly by the neutron vortices. The force acting on a unit length of a flux tube at the core-crustal interface is (Chau et al. 1992; Ding et al. 1993)

$$
f_{n}=\frac{2 \Phi_{0} \rho_{c} R_{c} \Omega_{s} \omega_{\mathrm{cr}}}{B_{c}},
$$

where $\Phi_{0}=h c / 2 e \sim 2 \times 10^{-7} \mathrm{G} \mathrm{cm}^{-2}$ is the flux quantum; the subscript $c$ represents the values in the core; $\Omega_{S}$ is the rotation rate of the core superfluid and can be approximated as that of the crust, $\Omega_{c}$, or the spin rate of the star in our following estimation; $\omega_{\mathrm{cr}}$ is the maximum angular velocity lag between $\Omega_{s}$ and $\Omega_{c}$; and $B_{c}$ is the core magnetic field, and could be only about $10^{-3}$ of the surface value, because the flux tubes are pushed out of the core due to spin-down (Ding et al. 1993). Thus, we can estimate the total magnitude of the force acting on the crust platelet with area $A$. The number of flux tubes anchored in this platelet is given by

$$
\begin{aligned}
N_{f} & =N_{\text {tot }} \frac{A}{\pi R_{c}^{2}} \\
& =\frac{A}{\pi R_{c}^{2}} \frac{\pi R_{c}^{2} B_{c}}{\Phi_{0}}=\frac{A B_{c}}{\Phi_{0}} .
\end{aligned}
$$

Substituting $f_{n}$ in equation (6), we have the total driving force on a platelet:

$$
F_{n}=N_{f} f_{n} R_{c}=2 A \rho_{c} R_{c}^{2} \Omega_{s} \omega_{\mathrm{cr}}
$$

We then make a dimension analysis to estimate the velocity

$$
\langle v\rangle \sim \sqrt{F / \rho A}=\left(R_{c}^{2} \Omega_{s} \omega_{\mathrm{cr}}\right)^{1 / 2} .
$$

Various models give $\omega_{\text {cr }}$ as being of order $10^{-6} \mathrm{rad} \mathrm{s}^{-1}$ (Chau et al. 1992); therefore, we have the velocity of the flux tube about $10^{3} \mathrm{~cm} \mathrm{~s}^{-1}$.

However, when the flux tubes move, they will experience a drag force by the electron sea in the core. The drag force per unit length of a single flux tube is given by

$$
f_{v}\left(v_{p}\right)=\frac{3 \pi}{64} \frac{n_{e} e^{2} \Phi_{0}^{2} v_{p}}{E_{f} \Lambda c},
$$

where $n_{e} \sim 10^{37} \mathrm{~cm}^{-3}$ is the electron density, and $E_{f}$ is the electron Fermi energy, which is about $100 \mathrm{MeV}$. The penetration length of a proton $\Lambda$ is $\sim 100 \mathrm{fm}$, and $v_{p}$ the velocity of the flux tube. By equating the vortex acting force $f_{n}$ and drag force $f_{v}$, we calculate the velocity as

$$
\begin{aligned}
v_{p} & =\frac{64}{3 \pi} \frac{E_{f} \Lambda c \rho_{c} R_{c} \Omega_{s} \omega_{\mathrm{cr}}}{B_{c} n_{e} e^{2} \Phi_{0}} \\
& \approx 10^{-12} \mathrm{~cm} \mathrm{~s}^{-1},
\end{aligned}
$$

and Ruderman et al. (1998) have considered the collective motion of flux tubes and gave an estimation of $10^{-6} \mathrm{~cm} \mathrm{~s}^{-1}$. If we equate the magnetic stress force $S A$ and the drag force by

$$
\frac{B B_{c}}{8 \pi} A=f_{v}\left(v_{p}\right) N_{f} R_{c}
$$

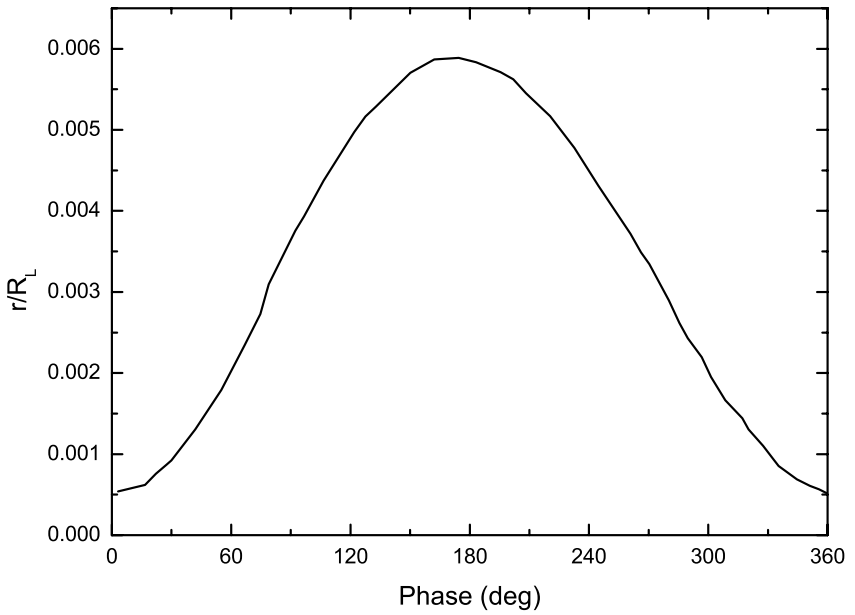

FIG. 6.- Variation of radial distance of the local position where the arrival photons are generated with the pulse phase in Fig. 4.

we have the velocity

$$
v_{p}=\frac{64}{24 \pi^{2}} \frac{B E_{f} \Lambda c}{n_{e} e^{2} \Phi_{0} R_{c}}
$$

at about $10^{-7}$ to $10^{-8} \mathrm{~cm} \mathrm{~s}^{-1}$. Therefore, we find the velocity of the crustal motion is too small to account for the emitting region drift.

On the other hand, if we consider the drift velocity of the plasma driven by the electric field in the magnetosphere, we can write

$$
v_{d}(D) \sim \frac{\delta \boldsymbol{E} \times \boldsymbol{B}}{B^{2}} c \sim\left|\frac{\delta E}{B(D)}\right| c,
$$

where $\delta E$ is the residual electric field which drives the plasma to move, and $B(D)$ is the local magnetic field at the radial distance $D$, where the emitting plasma is located. As shown in Figure 6, the average radial distance of the emitting region is about $D \sim$ $0.002 R_{L}=0.7 \times 10^{8} \mathrm{~cm}$. The length of the magnetic flux loop at $a_{1}=10$ is of order about $\mathcal{L} \sim 0.01 R_{L}$ (see Fig. 1). The residual electric field could be determined by $\delta E \approx \Delta V / \mathcal{L}$, where $\Delta V$ is the electric potential drop.

In closed field it is not clear how any substantial potential can survive, because electrons and positrons can be created and trapped in the closed field line region. These electron/positron pairs can screen the electric field. In our simulation, we find that the emission region is characterized by $a_{1}=10$, which is not far away from the open field line region. For a platelet with characteristic dimension of $\sim 10^{5} \mathrm{~cm}$, we can imagine that part of the platelet is the open field line region, where a characteristic potential $\Delta V \approx$ $6.6 \times 10^{15} B_{15} / P^{2}$ volts can be maintained (Ruderman \& Sutherland 1975). Here, $B_{15}$ is the surface magnetic field in unit of $10^{15} \mathrm{G}$. Thus, we have $\delta E \sim 1 \times 10^{3} B_{15}$ esu $\mathrm{cm}^{-2}$. Therefore, equation (14) could be rewritten as

$$
\begin{aligned}
v_{d} & \approx \frac{\delta E}{B_{s}\left(R_{*} / D\right)^{3}} c \\
& \approx 3 \times 10^{4} D_{8}^{3} \mathrm{~cm} \mathrm{~s}^{-1},
\end{aligned}
$$

where $D_{8}$ is the radial distance of the emitting region in unit of $10^{8} \mathrm{~cm}$. By substituting the average value $D_{8}=0.7$, the drift velocity of the emitting region is $v_{d}=10^{4} \mathrm{~cm} \mathrm{~s}^{-1}$, which is 


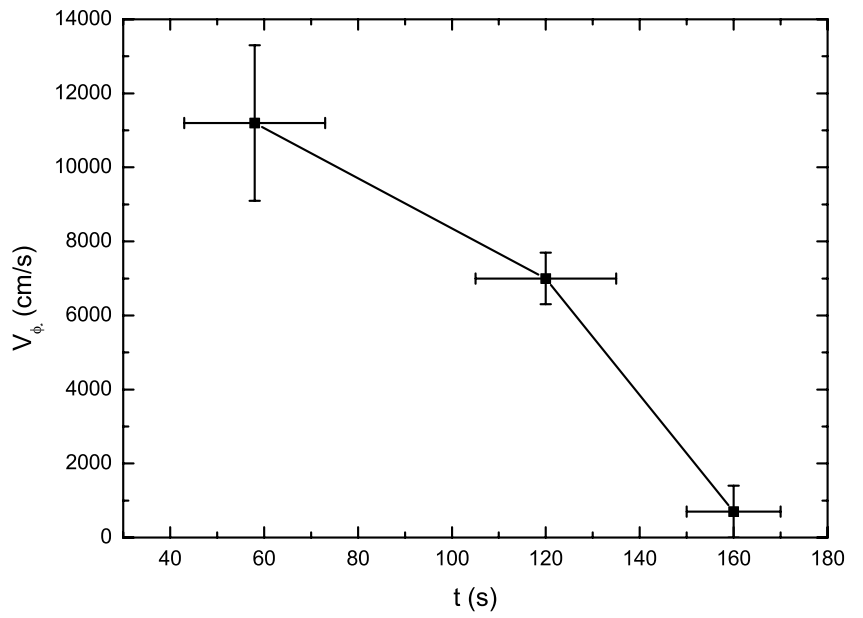

FIG. 7.- Speed of the emitting region drift in the $\phi_{*}$-direction is calculated by the comparison between our numerical results in Fig. 4 and the observation. The speed is given by $v_{\phi_{*}}\left[t=\left(t_{1}+t_{2}\right) / 2\right]=\Delta x / \Delta t$, where $\Delta x$ is the displacement of the emitting region centers in two adjacent panels in Fig. 4 , and $\Delta t=t_{2}-t_{1}$ is the time interval. Both $\Delta t$ and the error of $t$ are roughly determined from the timescale of Supplementary Fig. 1 in Palmer et al. (2005). The error of $v_{\phi_{*}}$ is calculated from the error propagation $\sigma\left(v_{\phi_{*}}\right) / \bar{v}_{\phi_{*}}=\sigma(t) / \bar{t}$. Since the emitting region changes the direction of movement or stops moving after $t=160 \mathrm{~s}$, we set $v_{\phi_{*}}(t=160 \mathrm{~s}) \approx 0$.

consistent with our result in Figure 7. Furthermore, as illustrated in equation (14), the drift velocity is proportional to the magnitude of the residual electric field, we may also argue that when $\delta E$ decays as the equilibrium charge distribution in the open field line region is being reestablished, the drift velocity becomes smaller. Even the emitting region could stop drifting and finally stay in the location indicated in Figure $4 c$, where it would emit the rest radiation. If such a case applies, it becomes unnecessary for us to propose the sudden change of motion direction in Figure 5.

\section{SUMMARY AND DISCUSSION}

We have investigated the timing properties of SGRs in the persistent state and the outburst tails, and described the variety of the pulsed radiation morphologies as the emissions coming from the closed field line regions inside the neutron star magnetosphere. For the weak relativistic effects in the magnetosphere closer to the stellar surface, the features of the light curve of SGRs that differed from those of Crab and Vela could be explained. Furthermore, by assuming the emitting region drift in the magnetosphere, we are able to simulate the pulse profile evolution of SGR 1806-20 during the giant flare on 2004 December 27. In addition, when we take the emissions from both magnetic poles into account, we are also able to explain the occurrence of the third peak, with the phase roughly consistent with the observation, which increases in strength relative to the two major ones (e.g., Fig. 2 in Palmer et al. 2005 ) at the end of the tails after the outburst. We believe that when the emission mechanism becomes clear, the emissivity on the field lines should not be uniform. In that case we may be able to predict a stronger third peak.

Feroci et al. (2001) made simple analytic fits to the flare light curves of SGR 1900+14 on 1998 August 27, and concluded that a fireball with contracting surface (rather than a cooling surface of fixed area) could provide a reasonable explanation for the decay tail of the outburst. They also proposed that the four-peaked profile was produced by several X-ray jets tied to the neutron star surface. However, the size of the radiation region in our fitting of SGR 1806-20 is unchanging, and the smooth decay in the tail phase of the giant flare might be due to the cooling of the plasma. It was believed that the phase stability of the pulses in light curve is due to the fixed location of the emitting region on the stellar surface. But our results show that a small migration could not break the stability in phase.

We have speculated some possible mechanisms, which cause the radiation region to migrate. It seems very clear that the physical motion of magnetic field lines, where the charged particles are trapped, must be very slow due to the extremely large drag force by the electrons in the core of neutron star. On the other hand, the $\boldsymbol{E} \times \boldsymbol{B}$ drift seems more possible. However, how this residual electric field survives from the screen of electron/positron pairs is not clear. We argue that one possible way is that some platelet is in the open field lines. It is unclear if this situation always occurs.

The 3-100 keV phase-averaged spectrum of the pulsed tail during the 2004 burst is fitted by a blackbody function at the temperature of $5.1 \mathrm{keV}$ plus a power law (Hurley et al. 2005). We did not give the calculated spectrum in this paper, for the radiation mechanism in the closed field lines needs further work. However, we argue that the power-law component results from the synchrotron radiation by the charged particles gyrating along the magnetic field lines. We need more information in the higher energy band and the phase-resolved spectra to provide more constrains and modification on our geometrical model.

We thank C. Thompson and M. Ruderman for useful discussions and the anonymous referee for helpful comments to improve the paper. This work is supported by a RGC grant of Hong Kong SAR Government, and Y. F. Huang is also supported by the National Natural Science Foundation of China (Grants 10625313 and 10221001).
Arendt, P. N., \& Eilek, J. A. 1998, preprint (astro-ph/9801257)

Chau, H. F., Cheng, K. S., \& Ding, K. Y. 1992, ApJ, 399, 213

Cheng, K. S., Ho, C., \& Ruderman, M. A. 1986, ApJ, 300, 500

Cheng, K. S., Ruderman, M. A., \& Zhang, L. 2000, ApJ, 537, 964

Chiang, J., \& Romani, R. W. 1994, ApJ, 436, 754

Daugherty, J. K., \& Harding, A. K. 1996, ApJ, 458, 278

Ding, K. Y., Cheng, K. S., \& Chau, H. F. 1993, ApJ, 408, 167

Dyks, J., \& Rudak, B. 2003, ApJ, 598, 1201

Fernández, R., \& Thompson, C. 2007, ApJ, 660, 615

Feroci, M., Hurley, K., Duncan, R. C., \& Thompson, C. 2001, ApJ, 549, 1021

Goldreich, P., \& Julian, W. H. 1969, ApJ, 157, 869

Harding, A. K. 1981, ApJ, 245, 267

Hurley, K., et al. 1999, Nature, 397, 41

. 2005, Nature, 434, 1098

Jia, J. J., Tang, A. P. S., Takata, J., Chang, H. K., \& Cheng, K. S. 2007, Adv. Space Res., 40, 1425

\section{REFERENCES}

Mazets, E. P., et al. 1979, Nature, 282, 587

Palmer, D. M., et al. 2005, Nature, 434, 1107

Romani, R. W., \& Yadigaroglu, I.-A. 1995, ApJ, 438, 314

Ruderman, M. 1991, ApJ, 382, 587

Ruderman, M., \& Sutherland, P. A. 1975, ApJ, 196, 51

Ruderman, M., Zhu, T. H., \& Chen, K. Y. 1998, ApJ, 492, 267

Thompson, C., \& Duncan, R. C. 1995, MNRAS, 275, 255

- 1996, ApJ, 473, 322

Thompson, C., Lyutikov, M., \& Kulkarni, S. R. 2002, ApJ, 574, 332

Woods, P. M., \& Thompson, C. 2006, in Compact Stellar X-Ray Sources, ed.

W. Lewin \& M. van der Klis (Cambridge: Cambridge Univ. Press), 547

Woods, P. M., et al. 2007, ApJ, 654, 470

Yadigaroglu, I.-A. 1997, Ph.D. thesis, Stanford Univ. 